\title{
Magnetic Resonance Imaging for Colorectal Cancer Metastasis to the Liver: Comparative Effectiveness Research for the Choice of Contrast Agents
}

Nieun Seo, MD, PhD'1
Mi-Suk Park, MD, PhD
Kyunghwa Han, $\mathrm{PhD}^{2}$
Kyung Ho Lee, MD, PhD
Seong Ho Park, MD, PhD'
Gi Hong Choi, MD, PhD
Jin-Young Choi, MD, $\mathrm{PhD}^{1}$
Yong Eun Chung, MD, PhD
Myeong-Jin Kim, MD, PhD

${ }^{1}$ Department of Radiology, Severance Hospital, Yonsei University College of Medicine, Seoul, ${ }^{2}$ Department of Radiology, Yonsei Biomedical Research Institute, Research Institute of Radiological Science, Seoul, ${ }^{3}$ Department of Radiology, Seoul National University Bundang Hospital, Seoul National University College of Medicine, Seongnam, ${ }^{4}$ Department of Radiology and Research Institute of Radiology, Asan Medical Center, University of Ulsan College of Medicine, Seoul, ${ }^{5}$ Department of Surgery, Severance Hospital, Yonsei University College of Medicine, Seoul, Korea
Correspondence: Mi-Suk Park, MD, PhD

Department of Radiology, Severance Hospital, Yonsei University College of Medicine, 50-1 Yonsei-ro, Seodaemun-gu,

Seoul 03722, Korea

Tel: $82-2-2228-7400$

Fax: 82-2-393-3035

E-mail: radpms@yuhs.ac

Received November 8, 2016

Accepted February 19, 2017

Published Online March 14, 2017

\section{Purpose}

This study was conducted to compare the diagnostic performance and early recurrence rate between gadoxetic acid-enhanced magnetic resonance imaging (Gd-EOB-MRI) and magnetic resonance imaging (MRI) with extracellular contrast agent (ECA-MRI) for evaluating hepatic lesions in colorectal cancer.

\section{Materials and Methods}

Between 2005 and 2010, 418 colorectal cancer patients with both preoperative computed tomography (CT) and liver MRI were retrospectively reviewed. Image analysis was based on initial radiologic reports, and diagnostic performance was assessed based on the area under the receiver operating characteristic curve (AUROC). The early intrahepatic recurrence rate within 6 months was then evaluated.

\section{Results}

Overall, 291 and 127 patients underwent Gd-EOB-MRI and ECA-MRI, respectively. The AUROCs were not significantly different between Gd-EOB-MRI (0.990; 95\% Cl, 0.980 to $0.999)$ and ECA-MRI $(0.985 ; 95 \% \mathrm{Cl}, 0.968$ to $1.000 ; \mathrm{p}=0.836)$. When compared with CT alone, ECA-MRI detected additional 21 lesions in 14 patients (14/127, 11.0\%), whereas Gd-EOB-MRI detected 56 lesions in 33 patients (33/291, 11.3\%) without a significant difference between two MRI groups $(p=0.331)$. The early recurrence rate in the ECA-MRI (28.6\%) was significantly higher than that in the Gd-EOB-MRI (11.6\%) for patients who underwent hepatic resection $(p=0.031)$.

\section{Conclusion}

Gd-EOB-MRI is potentially better than ECA-MRI for decreasing the early intrahepatic recurrence rate, although the two MRI modalities showed comparable diagnostic performance in colorectal cancer patients.

\section{Key words}

Colorectal neoplasms, Liver, Neoplasm metastasis,

Magnetic resonance imaging, Recurrence 


\section{Introduction}

The liver is the most common organ of distant metastasis in colorectal cancer, and approximately $15 \%-20 \%$ of patients present with liver metastasis at the initial staging [1,2] Because colorectal liver metastasis (CRLM) is an important prognostic factor and surgical resection can improve survival in suitable patients $[3,4]$, accurate staging is essential for the proper management of and improvement of survival in these patients. Most colorectal cancer patients undergo computed tomography (CT) for evaluation of the initial disease status, including that of the liver [5-7]. However, CT has inherent limitations when applied for detecting and characterizing small hepatic lesions $(<1 \mathrm{~cm})$, as well as for detecting hepatic lesions in the fatty liver $[7,8]$.

The majority of the current clinical guidelines, including the National Comprehensive Cancer Network guidelines, do not necessitate the use of magnetic resonance imaging (MRI) for evaluation of CRLM. However, MRI is a widely used problem-solving tool during evaluation of the liver in colorectal cancer. MRI not only increases the confidence in diagnosing small indeterminate lesions detected on CT $[9,10]$ but also detects additional CRLM, especially for lesions $<1 \mathrm{~cm}$ [11-13]. After its initial approval in Europe in 2004, gadoxetic acid (Primovist, Bayer Schering Pharma, Berlin, Germany; Eovist, Bayer Healthcare Pharmaceuticals, Whippany, NJ) was approved in many Asian countries at 2007 and the United States at 2008, and has since been widely applied for liver MRI. Many studies have shown that gadoxetic acidenhanced MRI (Gd-EOB-MRI) is superior to CT, and recent consensus guidelines from the radiologic community recommend Gd-EOB-MRI for the preoperative evaluation of CRLM [14,15]. Gadoxetic acid has different pharmacokinetic and pharmacodynamic properties from extracellular contrast agents. Specifically, gadoxetic acid has the combined properties of an extracellular contrast agent during the early vascular-interstitial phase and a liver-specific agent during the delayed phase [16]. Because of the different properties and variations in cost of contrast agents, evidence-based information is needed to enable decision making regarding which contrast agent should be used in patients with CRLM.

To the best of our knowledge, there is no solid evidence of the clinical benefits of Gd-EOB-MRI over MRI with an extracellular contrast agent (ECA-MRI) in this setting. Therefore, we retrospectively compared the effectiveness of Gd-EOBMRI and ECA-MRI in CRLM in terms of diagnostic performance and early intrahepatic recurrence rate.

\section{Materials and Methods}

\section{Patients}

This retrospective cohort study was approved by our institutional review board, and the need for informed consent was waived. Fig. 1 shows the flow of patient enrollment. We searched the electronic medical records and found 3,549 patients who presented with newly diagnosed colorectal cancer between January 2005 and December 2010. The study period was selected to include patients who underwent Gd-EOB-MRI or ECA-MRI and had sufficient follow-up periods. Among these patients, 505 were excluded for the following reasons: (1) past or concurrent malignancy other than colorectal cancer $(n=308)$; $(2)$ absence of operation for primary colorectal cancer ( $\mathrm{n}=147$ ); and (3) no contrast-enhanced abdominopelvic CT prior to any treatment $(n=50)$. Among the remaining patients, 2,516 without contrast-enhanced liver MRI before treatment and 110 who met the following criteria were also excluded: (1) MRI contrast agent other than an extracellular agent or gadoxetic acid ( $\mathrm{n}=38)$; (2) multiple hepatic lesions (>10) on CT ( $\mathrm{n}=23)$; (3) distant metastasis to sites other than the liver $(n=20)$; (4) interval between CT and liver MRI of $>4$ weeks ( $n=24)$; and (5) unavailable reference standard for hepatic lesions ( $n=5)$. Finally, 418 colorectal cancer patients who underwent both abdominopelvic CT and liver MRI before treatment were included. Demographic information including age, sex, serum carcinoembryonic antigen (CEA) level, pathologic tumor node metastasis staging system, American Joint Committee on Cancer staging, location of the primary tumor, and differentiation of the primary tumor was reviewed, as well as whether liver operation was performed. For patients who had undergone hepatic resection, the presence or absence of adjuvant chemotherapy or radiotherapy was investigated.

\section{CT and MRI acquisition}

At our institution, liver MRI is generally performed for patients found to have potentially resectable liver metastases on CT, according to the National Comprehensive Cancer Network guidelines [5,6]. Liver MRI is also performed for patients with an indeterminate lesion on CT or for high-risk patients, at the discretion of surgeons and physicians.

All CT scans were performed with a 16-, 64-, or 128-channel multidetector (Siemens Healthcare, Erlangen, Germany; GE Healthcare, Waukesha, WI). Portal-phase abdominopelvic CT was obtained 70 seconds after the intravenous administration of $2.0 \mathrm{~mL} / \mathrm{kg}$ nonionic contrast material (iopromide, Ultravist 300, Bayer Schering Pharma), followed by a $20 \mathrm{~mL}$ saline chaser bolus injection at a fixed duration 


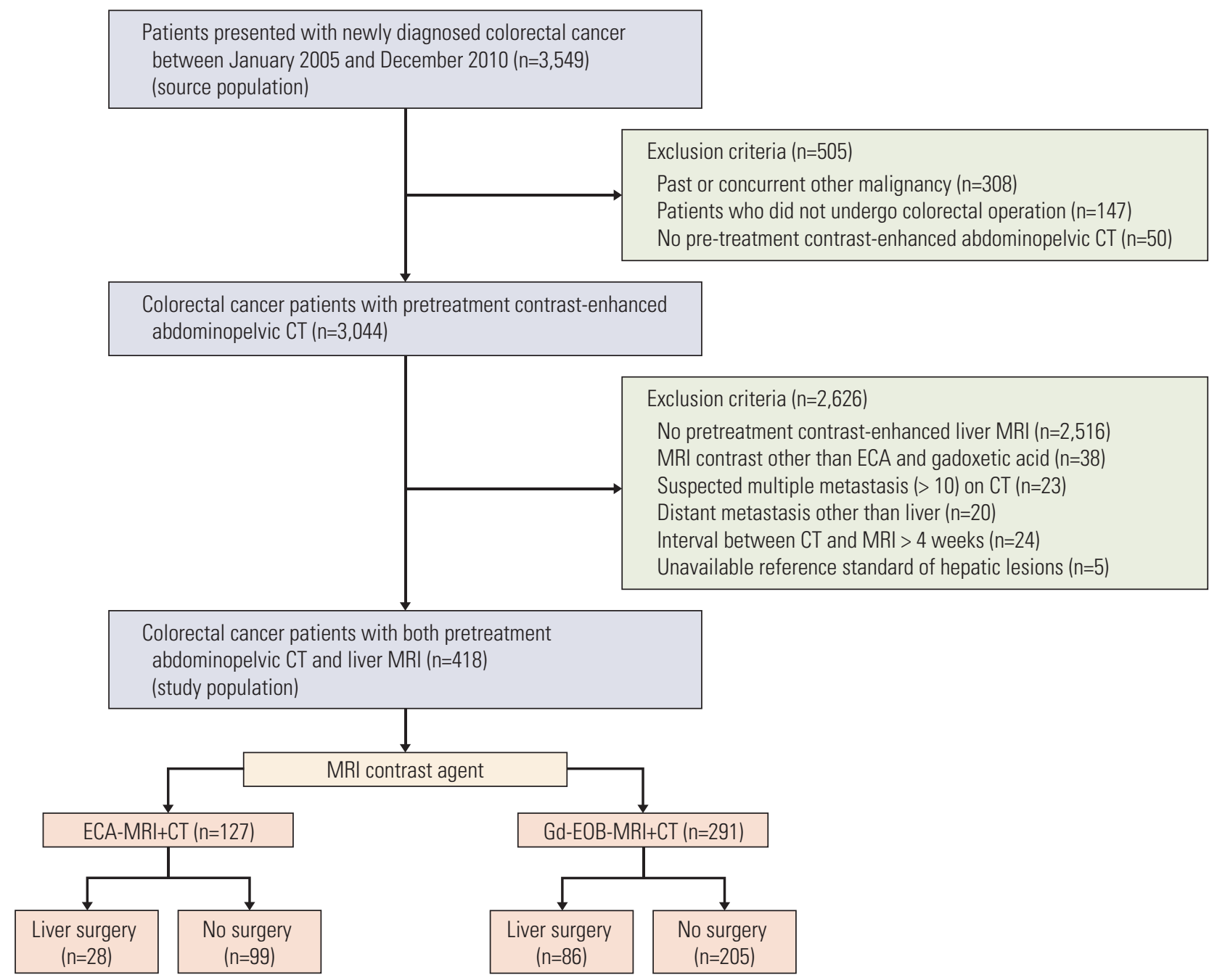

Fig. 1. Flow of patient enrollment. CT, computed tomography; MRI, magnetic resonance imaging; ECA, extracellular contrast agent; ECA-MRI, magnetic resonance imaging with extracellular contrast agent; Gd-EOB-MRI, gadoxetic acid-enhanced magnetic resonance imaging.

of 30 seconds.

Liver MRI was performed with a 1.5-T (Philips Healthcare, the Best, the Netherlands) or 3.0-T magnet (Siemens Healthcare, Philips Healthcare). Magnetic resonance (MR) sequences that were similar for ECA-MRI and Gd-EOB-MRI included dual-echo in-phase and opposed-phase spoiled gradient-echo T1-wei-ghted images, as well as multishot and single-shot turbo spin-echo T2-weighted images. Dynamic fat-suppressed spoiled gradient-echo T1-weighted images were acquired before and after contrast material injection (arterial, portal venous, 3-minute delayed, and 5-minute delayed phases). For ECA-MRI, variable gadolinium-based contrast agents, including gadopentetate dimeglumine (Mag- nevist, Bayer Schering Pharma) and gadoterate meglumine (Dotarem, Guerbet, Roissy, France), were used, and 0.1 $\mathrm{mmol} / \mathrm{kg}$ gadolinium agent was administered intravenously. For dynamic scan with gadoxetic acid disodium (Primovist, Bayer Schering Pharma), $0.1 \mathrm{~mL} / \mathrm{kg}(0.025 \mathrm{mmol} /$ $\mathrm{kg}$ ) gadoxetic acid disodium was injected, and additional hepatobiliary phase images were obtained after 15 or 20 minutes. Gadoxetic acid has replaced extracellular contrast agents for liver MRI at our institution since January 2008; therefore, the decision to perform ECA-MRI or Gd-EOB-MRI was not influenced by the patient or physician factor, but by the study period. 


\section{Image analysis}

The CT and MRI images were prospectively interpreted as the routine practice using a picture archiving and communication system by six board-certified abdominal radiologists with 7 to 20 years of experience in liver imaging. Each focal hepatic lesion was classified as follows: 1, definitely benign; 2 , probably benign; 3 , indeterminate; 4 , probably metastatic; and 5, definitely metastatic. Category 1 included typical benign lesions such as cyst, hemangioma, or calcification. Category 2 included lesions that were judged to be more likely benign than metastatic but the benignity was not definitive, whereas category 4 represented the opposite situation. Category 3 included tiny hepatic lesions or lesions that were difficult to categorize as either benign or metastatic. Category 5 was assigned to typical metastatic lesions. Given this retrospective study design, a study coordinator (N.S., a board-certified abdominal radiologist with a 3-year non-trainee experience in liver imaging) rescored the lesion category based on clinical radiologic reports. The study coordinator also reviewed the size, segmental location, and number of the hepatic lesions on the basis of the initial radiologic reports. Additional lesions detected on liver MRI compared with CT alone were recorded in the same manner. Only additional lesions of MRI category 3-5 were recorded, while additionally detected definitely or probably benign lesions were not recorded.

\section{Confirmation of hepatic lesions}

The study coordinator assessed the hepatic outcomes. All patients underwent surgery for primary colorectal cancer. During the operation, the liver was assessed by manual palpation and often with intraoperative ultrasonography (IOUS) for suspected liver metastasis. IOUS was performed by board-certified radiologists in 15.7\% (20 of 127) of the ECAMRI group and 16.5\% (69 of 291) of the Gd-EOB-MRI group. In patients for which there were no pathological results for hepatic lesions, hepatic outcome was determined through follow-up imaging. On follow-up imaging, increase or decrease in the size of the hepatic lesions during chemotherapy was considered metastasis. In contrast, the decrease in size or disappearance of the hepatic lesion without treatment was considered as benign. Lesions that remained stable for at least 12 months were also considered benign.

\section{Follow-up}

At our institution, patients underwent physical examinations and measurement of CEA at 3-month intervals over 2 years, then at 6-month intervals during the next 3 years. Contrast-enhanced abdominopelvic CT and chest CT were per- formed at 6- and 12-month intervals, respectively, during the first 5 years after surgery. In patients with resected metastasis, contrast-enhanced abdominopelvic CT and chest CT were performed at shorter intervals of 3 and 6 months, respectively, during the first 2 years after surgery, then every 6 months up to a total 5 years. The dates of last follow-up and intrahepatic recurrence were recorded.

\section{Statistical analysis}

The data were analyzed in two separate groups. First, the diagnostic performance for focal hepatic lesions was evaluated in all study patients, and early intrahepatic recurrence was evaluated for patients who were initially classified as not having hepatic metastasis on preoperative imaging. Second, the diagnostic performance and early intrahepatic recurrence were assessed in patients who underwent curative hepatic resection for suspected metastases.

The baseline demographics and characteristics of hepatic lesions in the ECA-MRI and Gd-EOB-MRI groups were compared by independent $t$ tests for continuous variables, and by Pearson's chi-squared or Fisher exact test for categorical variables. The diagnostic performance of MRI for predicting liver metastasis was evaluated through receiver operating characteristic (ROC) analysis. The area under the ROC curve (AUROC) was reported with 95\% confidence intervals (CIs). Per-lesion and per-patient analyses were conducted. For the per-lesion analysis, subgroup analysis was conducted for small hepatic lesions $\leq 1 \mathrm{~cm}$ on MRI. The AUROC was compared between ECA-MRI and Gd-EOB-MRI using chisquared statistics. The number and size of nodules that were additionally detected on MRI compared to CT were compared by the Mann-Whitney $U$ test and independent $t$ test, respectively.

The early intrahepatic recurrence rate was evaluated through a competing risk analysis. Death of any cause was considered a competing event, as death precludes the observation of recurrence. Early intrahepatic recurrence was defined as intrahepatic recurrence within 6 months of curative hepatic resection. For patients who did not undergo hepatic resection, early intrahepatic recurrence was defined as recurrence within 6 months of colorectal surgery. The differences in the cumulative incidence of tumor recurrence between ECA-MRI and Gd-EOB-MRI were compared by Gray's test [17]. Univariate and multivariate analyses were performed with Fine and Gray's subdistributional hazard regression model to identify independent prognostic factors of early recurrence [18]. Multivariate analysis was performed for factors with a p-value of $<0.1$ on univariate analysis, and clinically important factors ( $\mathrm{N}$ stage, largest size and number of metastasis, and type of MRI contrast agent). All statistical analyses were conducted using SAS ver. 9.2 (SAS Institute 
Table 1. Baseline characteristics of study population

\begin{tabular}{|c|c|c|c|}
\hline Variable & ECA-MRI (n=127) & Gd-EOB-MRI $(n=291)$ & p-value \\
\hline Age (yr) & $60.0 \pm 11.5$ & $61.2 \pm 11.4$ & 0.33 \\
\hline Sex (male:female) & $67(52.8): 60(47.2)$ & 171 (58.8):120 (41.2) & 0.254 \\
\hline CEA (ng/dL) & 13.9 & 19.81 & 0.373 \\
\hline \multicolumn{4}{|l|}{ T stage } \\
\hline $\mathrm{T} 1$ & $5(3.9)$ & $19(6.5)$ & 0.516 \\
\hline T2 & $12(9.4)$ & $31(10.7)$ & \\
\hline T3 & $88(69.3)$ & $203(69.8)$ & \\
\hline $\mathrm{T} 4$ & $22(17.3)$ & $38(13.1)$ & \\
\hline \multicolumn{4}{|l|}{$\mathrm{N}$ stage } \\
\hline N0 & $57(44.9)$ & $200(47.8)$ & 0.71 \\
\hline N1 & $43(33.9)$ & $136(32.5)$ & \\
\hline N2 & $27(21.3)$ & $82(19.6)$ & \\
\hline \multicolumn{4}{|l|}{ M stage } \\
\hline M0 & $87(68.5)$ & $296(70.8)$ & 0.493 \\
\hline M1 & $40(31.5)$ & $122(29.2)$ & \\
\hline \multicolumn{4}{|l|}{ AJCC stage } \\
\hline I & $13(10.2)$ & $50(12.0)$ & 0.790 \\
\hline II & $34(26.8)$ & $119(28.5)$ & \\
\hline III & $40(31.5)$ & $127(30.4)$ & \\
\hline IV & $40(31.5)$ & $122(29.2)$ & \\
\hline \multicolumn{4}{|l|}{ Location of CRC } \\
\hline Colon & $82(64.6)$ & $180(61.9)$ & 0.598 \\
\hline Rectum & $45(35.4)$ & 111 (38.1) & \\
\hline \multicolumn{4}{|l|}{ Differentiation } \\
\hline Well & $27(21.3)$ & $37(12.7)$ & 0.068 \\
\hline Moderately & $90(70.9)$ & $234(80.4)$ & \\
\hline Poorly or mucinous & $10(7.8)$ & $20(6.9)$ & \\
\hline \multicolumn{4}{|l|}{ Liver surgery } \\
\hline Yes & $28(22.0)$ & $86(29.6)$ & 0.098 \\
\hline No & $99(78.0)$ & $205(70.4)$ & \\
\hline
\end{tabular}

Values are presented as mean \pm standard deviation or number (\%). ECA-MRI, magnetic resonance imaging with extracellular contrast agent; Gd-EOB-MRI, gadoxetic acid-enhanced magnetic resonance imaging; CEA, carcinoembryonic antigen; AJCC, American Joint Committee on Cancer; CRC, colorectal cancer.

Inc., Cary, NC), and a $\mathrm{p}<0.05$ was considered statistically significant.

\section{Results}

\section{Analysis of hepatic lesions from all patients}

\section{1) Patients}

Of the 418 total patients, 127 (male:female [M:F], 67:60; mean age \pm standard deviation [SD], $60 \pm 11.5$ years) underwent ECA-
MRI and 291 (M:F, 171:120; 61.2 \pm 11.4 years) underwent Gd-EOB-MRI. No demographic data differed significantly between the ECA-MRI and Gd-EOB-MRI groups (Table 1). Overall, 22\% (28 of 127) and $29.6 \%$ (86 of 291) of patients in the ECA-MRI and Gd-EOB-MRI groups underwent liver resection, respectively. The median time interval between $\mathrm{CT}$ and liver MRI was 6 days (range, 0 to 28 days).

\section{2) Characterization and proof of hepatic lesions}

A total of 635 hepatic lesions in 418 patients were confirmed pathologically $(n=285)$ or on follow-up imaging $(n=350)$. For pathologically confirmed lesions, the median time interval between MRI and biopsy or surgery was 8 days (range, 0 to 
Table 2. Characteristics of hepatic lesions evaluated on liver MRI

\begin{tabular}{|c|c|c|c|}
\hline Variable & ECA-MRI & Gd-EOB-MRI & p-value \\
\hline \multicolumn{4}{|l|}{ Per-patient analysis } \\
\hline No. of patients & 127 & 291 & \\
\hline Benign:Metastatic & 85 (66.9):42 (33.1) & $202(69.4): 89(30.6)$ & 0.614 \\
\hline The largest size of lesion $(\mathrm{cm})$ & $2.4 \pm 1.8$ & $2.3 \pm 1.7$ & 0.408 \\
\hline No. of lesions & $1.7 \pm 1.0$ & $1.3 \pm 0.8$ & 0.102 \\
\hline \multicolumn{4}{|l|}{ Per-lesion analysis } \\
\hline No. of lesions & 205 & 430 & \\
\hline Benign:Metastatic & 111 (54.1):94 (45.9) & $226(52.6): 204(47.4)$ & 0.772 \\
\hline Size of lesion, total (cm) & $1.2 \pm 1.4$ & $1.2 \pm 1.2$ & 0.676 \\
\hline Benign $(\mathrm{cm})$ & $0.7 \pm 0.7$ & $0.8 \pm 0.7$ & 0.136 \\
\hline Metastatic $(\mathrm{cm})$ & $1.8 \pm 1.7$ & $1.7 \pm 1.5$ & 0.722 \\
\hline No. of lesions $\leq 1 \mathrm{~cm}(\%)$ & $118 / 205(57.6)$ & $227 / 430(52.8)$ & 0.259 \\
\hline
\end{tabular}

Values are presented as number or mean \pm standard deviation. MRI, magnetic resonance imaging; ECA-MRI, MRI with extracellular contrast agent; Gd-EOB-MRI, gadoxetic acid-enhanced MRI.

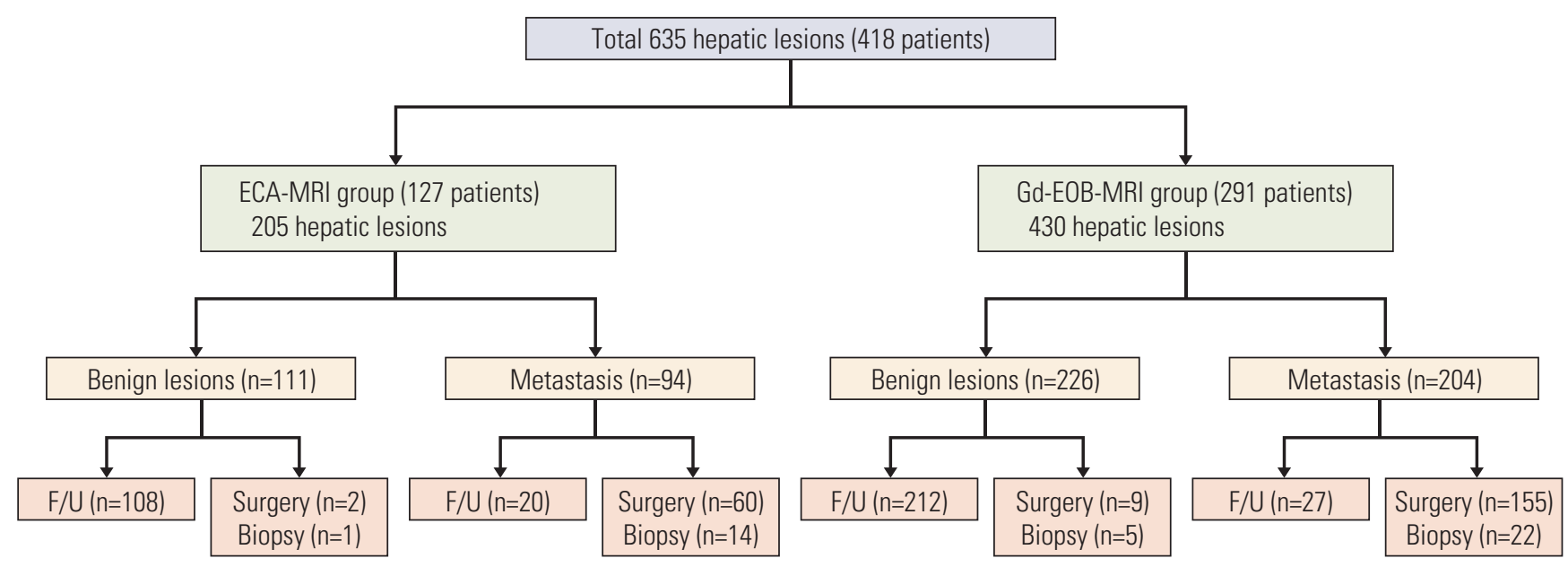

Fig. 2. Hepatic outcomes in study patients. This figure shows the true hepatic outcomes and methods of lesion confirmation in study patients. Hepatic lesions were confirmed by surgery, biopsy, or follow-up imaging (F/U). ECA-MRI, magnetic resonance imaging with extracellular contrast agent; Gd-EOB-MRI, gadoxetic acid-enhanced magnetic resonance imaging.

29 days). Table 2 shows the characteristics of hepatic lesions evaluated on ECA-MRI and Gd-EOB-MRI. The diameter of the largest lesion and mean lesion number per patient did not differ significantly between the ECA-MRI and Gd-EOB-MRI groups ( $\mathrm{p}=0.408$ and $\mathrm{p}=0.102$, respectively). The true hepatic outcome and methods of lesion confirmation are demonstrated in Fig. 2. Most benign lesions in the ECA-MRI (97.3\%, 108 of 111) and Gd-EOB-MRI (93.8\%, 212 of 226) groups were confirmed on follow-up imaging, with a median follow-up period of 49 months (range, 13 to 97 months) and 55 months (range, 16 to 84 months), respectively.

\section{3) Diagnostic performance}

For per-patient analysis, the AUROC of ECA-MRI (0.985; $95 \% \mathrm{CI}, 0.968$ to 1.000$)$ was not significantly different from that of Gd-EOB-MRI (0.990; 95\% CI, 0.980 to 0.999) ( $\mathrm{p}=0.836)$. For per-lesion analysis of the total 635 lesions, the AUROC 
Table 3. Additional lesions detected on liver MRI compared to CT

\begin{tabular}{lcccc} 
Variable & ECA-MRI & Gd-EOB-MRI & Total & p-value \\
Per-patient analysis & & & & \\
$\quad$ No. of patients (\%) & $14 / 127(11.0)$ & $33 / 291(11.3)$ & $47 / 418(11.2)$ & 0.331 \\
$\quad$ No. of lesions, median (range) & $1(1-4)$ & $1(1-6)$ & $1(1-6)$ & 0.530 \\
Per-lesion analysis & $21 / 205(10.2)$ & $56 / 430(13.0)$ & $77 / 635(12.1)$ & 0.316 \\
$\quad$ No. of additional lesions (\%) & $0.7 \pm 0.3$ & $0.6 \pm 0.4$ & $0.7 \pm 0.7$ & 0.495 \\
\hline Size of additional lesions, mean \pm SD (cm) &
\end{tabular}

MRI, magnetic resonance imaging; CT, computed tomography; ECA-MRI, MRI with extracellular contrast agent; Gd-EOB-

MRI, gadoxetic acid-enhanced MRI; SD, standard deviation.

Table 4. Baseline characteristics of patients who underwent liver surgery

\begin{tabular}{|c|c|c|c|}
\hline Variable & ECA-MRI (n=28) & Gd-EOB-MRI $(\mathrm{n}=86)$ & p-value \\
\hline Age (yr) & $57.4 \pm 11.2$ & $58.8 \pm 11.9$ & 0.602 \\
\hline Sex (male:female) & 20 (71.4):8 (28.6) & 51 (59.3):35 (40.7) & 0.250 \\
\hline \multicolumn{4}{|l|}{ CEA (ng/dL) } \\
\hline$\leq 5$ & $17(60.7)$ & $49(57)$ & 0.728 \\
\hline$>5$ & $11(39.3)$ & $37(43)$ & \\
\hline \multicolumn{4}{|l|}{ T stage } \\
\hline $\mathrm{T} 1$ & 0 & $4(4.7)$ & 0.127 \\
\hline T2 & 0 & $3(3.5)$ & \\
\hline T3 & $26(92.9)$ & $60(69.8)$ & \\
\hline $\mathrm{T} 4$ & $2(7.1)$ & $19(22.1)$ & \\
\hline \multicolumn{4}{|l|}{$\mathrm{N}$ status } \\
\hline Negative & $8(28.6)$ & $24(27.9)$ & $>0.999$ \\
\hline Positive & $20(71.4)$ & $62(72.1)$ & \\
\hline \multicolumn{4}{|l|}{ Location of CRC } \\
\hline Colon & $19(67.9)$ & $57(66.3)$ & 0.878 \\
\hline Rectum & $9(32.1)$ & $29(33.7)$ & \\
\hline \multicolumn{4}{|l|}{ Differentiation } \\
\hline Well & $3(10.7)$ & $11(12.8)$ & 0.626 \\
\hline Moderately & $24(85.7)$ & $66(76.7)$ & \\
\hline Poorly or mucinous & $1(3.6)$ & $9(10.5)$ & \\
\hline \multicolumn{4}{|c|}{ The largest size of lesion $(\mathrm{cm})$} \\
\hline Mean \pm SD & $2.2 \pm 1.9$ & $2.3 \pm 1.7$ & 0.850 \\
\hline$\leq 5$ & $27(96.4)$ & $80(93.0)$ & $>0.999$ \\
\hline$>5$ & $1(3.6)$ & $6(7.0)$ & \\
\hline \multicolumn{4}{|l|}{ No. of lesions } \\
\hline Mean \pm SD & $2.6 \pm 1.8$ & $2.2 \pm 1.5$ & 0.218 \\
\hline 1 & $8(28.6)$ & $45(52.3)$ & 0.029 \\
\hline$>1$ & $20(71.4)$ & $41(47.7)$ & \\
\hline \multicolumn{4}{|l|}{ Adjuvant chemotherapy } \\
\hline Yes & $27(96.4)$ & $80(93.0)$ & $>0.999$ \\
\hline No & $1(3.6)$ & $6(7.0)$ & \\
\hline \multicolumn{4}{|l|}{ Adjuvant radiotherapy } \\
\hline Yes & $7(25.0)$ & $20(23.3)$ & 0.850 \\
\hline No & $21(75.0)$ & $66(76.7)$ & \\
\hline
\end{tabular}

Values are presented as mean \pm standard deviation or number (\%). ECA-MRI, magnetic resonance imaging with extracellular contrast agent; Gd-EOB-MRI, gadoxetic acid-enhanced magnetic resonance imaging; CEA, carcinoembryonic antigen; CRC, colorectal cancer; SD, standard deviation. 
Table 5. Univariate and multivariate analysis of factors associated with early recurrence

\begin{tabular}{|c|c|c|c|c|}
\hline \multirow{2}{*}{ Variable } & \multicolumn{2}{|c|}{ Univariate } & \multicolumn{2}{|c|}{ Multivariate } \\
\hline & SDHR $(95 \% \mathrm{CI})$ & p-value & SDHR $(95 \% \mathrm{CI})$ & p-value \\
\hline Age & $1.020(0.977-1.060)$ & 0.430 & - & - \\
\hline Sex (male vs. female) & $2.310(0.782-6.840)$ & 0.130 & - & - \\
\hline CEA & $1.000(0.998-1.010)$ & 0.220 & - & - \\
\hline \multicolumn{5}{|l|}{ T stage } \\
\hline T2 vs. T1 & $1.282(0.082-20.040)$ & 0.860 & - & - \\
\hline T3 vs. T1 & $0.547(0.066-4.510)$ & 0.570 & - & - \\
\hline T4 vs. T1 & $0.481(0.049-4.770)$ & 0.530 & - & - \\
\hline \multicolumn{5}{|l|}{ N stage } \\
\hline N0 vs. N1-2 & $3.390(0.798-14.400)$ & 0.098 & $3.813(0.830-17.511)$ & 0.085 \\
\hline \multicolumn{5}{|l|}{ Location of CRC } \\
\hline Colon vs. rectum & $1.330(0.478-3.700)$ & 0.590 & - & - \\
\hline \multicolumn{5}{|l|}{ MRI contrast agent } \\
\hline Gd-EOB vs. ECA & $0.365(0.146-0.915)$ & 0.032 & $0.288(0.110-0.757)$ & 0.012 \\
\hline The largest size of lesion & $0.851(0.627-1.160)$ & 0.300 & $0.888(0.658-1.200)$ & 0.440 \\
\hline \multicolumn{5}{|l|}{ Lesion number per patient } \\
\hline 1 vs. $>1$ & $0.682(0.271-1.720)$ & 0.420 & $0.698(0.482-1.012)$ & 0.058 \\
\hline Chemotherapy & $1.070(0.131-8.630)$ & 0.950 & - & - \\
\hline Radiotherapy & $1.250(0.453-3.440)$ & 0.670 & - & - \\
\hline
\end{tabular}

SDHR, subdistributional hazard ratio; CI, confidence interval; CEA, carcinoembryonic antigen; CRC, colorectal cancer; MRI, magnetic resonance imaging; Gd-EOB, gadoxetic acid; ECA, extracellular contrast agent.

between ECA-MRI (0.980; 95\% CI, 0.963 to 0.997). and Gd-EOB-MRI (0.988; 95\% CI, 0.976 to 0.999$)$ was not significantly different $(\mathrm{p}=0.476)$. The AUROCs were also comparable between ECA-MRI (0.975; 95\% CI, 0.935 to 1.000) and Gd-EOBMRI (0.985; 95\% CI, 0.956 to 1.000$)(p=0.695)$ for the 345 lesions $\leq 1 \mathrm{~cm}$.

\section{4) Detection of additional nodules on MRI compared with CT}

In 47 patients $(11.2 \%, 47$ of 418$), 77$ lesions were additionally detected on liver MRI compared with CT (Table 3). ECA-MRI detected an additional 21 lesions in 14 patients, whereas Gd-EOB-MRI identified 56 additional lesions in 33 patients, without a significant difference between two MRI groups $(p=0.331)$. Among the 21 additional lesions on ECA-MRI, 17 were finally considered metastatic, while four were benign. Of the 56 additional lesions on Gd-EOB-MRI, 48 were metastatic and eight were benign.

\section{5) Early intrahepatic recurrence in patients considered not} to have hepatic metastasis

Eighty-five patients with ECA-MRI and 202 patients with Gd-EOB-MRI were initially considered to have only benign hepatic lesions on preoperative imaging. Among these patients, one in each MRI group developed early intrahepatic recurrence. For the ECA-MRI group, one patient $(1 / 85,1.2 \%)$ who had hepatic cysts on preoperative imaging developed hepatic, lymph nodes, and bone metastases at 6 months after primary colorectal surgery. In the Gd-EOB-MRI group, one patient $(1 / 202,0.5 \%)$ who had a hepatic cyst on preoperative imaging developed hepatic and lung metastases at 4 months after colorectal surgery. Comparison of the intrahepatic recurrence rate and analysis of independent predictors for intrahepatic recurrence between the two MRI groups could not be performed due to the small number of positive events.

\section{Analysis of patients who underwent hepatic surgery}

\section{1) Patients}

Among 114 patients who underwent curative liver surgery for suspected hepatic metastases, 28 (M:F, 20:8; mean age \pm SD, $57.4 \pm 11.2$ years) underwent ECA-MRI and 86 (M:F, 51:35; $58.8 \pm 11.9$ years) underwent Gd-EOB-MRI. Although most demographic variables were not significantly different between groups (Table 4), the number of patients who had more than one hepatic metastases was significantly greater in the ECA-MRI than the Gd-EOB-MRI group ( $\mathrm{p}=0.029$ ). 


\section{2) Diagnostic performance}

The 114 patients who underwent liver surgery had 260 focal hepatic lesions. For per-lesion analysis, the AUROC between ECA-MRI (0.924; 95\% CI, 0.818 to 1.000) and Gd-EOB-MRI (0.914; $95 \%$ CI, 0.825 to 1.000$)$ was not significantly different ( $\mathrm{p}=0.885$ ). For the 99 lesions $\leq 1 \mathrm{~cm}$, the AUROCs between ECA-MRI (0.895; 95\% CI, 0.752 to 1.000) and Gd-EOB-MRI (0.894; $95 \%$ CI, 0.805 to 0.984$)$ did not differ significantly $(\mathrm{p}=0.993)$.

\section{3) Early intrahepatic recurrence}

The cumulative incidence of early intrahepatic recurrence was significantly higher $(\mathrm{p}=0.031)$ in the ECA-MRI $(28.6 \%)$ than the Gd-EOB-MRI group (11.6\%). The results of univariate and multivariate analyses of factors associated with early intrahepatic recurrence are shown in Table 5. Differentiation of the primary colorectal tumor was not included in the univariate and multivariate analyses because of inhomogeneous data distribution. The type of contrast agent (ECA vs. Gd-EOB) was the only significant variable predicting early intrahepatic recurrence on univariate analysis (subdistributional hazard ratio [SDHR], 0.365; 95\% CI, 0.146 to 0.915 ) $(\mathrm{p}=0.032)$. Multivariate analysis also revealed that the use of Gd-EOB-MRI was a good prognostic factor (SDHR, 0.288; $95 \%$ CI, 0.110 to 0.757$)(\mathrm{p}=0.012)$.

\section{Discussion}

The results of this study demonstrated that the overall diagnostic performance of ECA-MRI and Gd-EOB-MRI for the evaluation of focal hepatic lesions in colorectal cancer before treatment did not differ significantly. In addition, the detection rate of additional significant hepatic lesions on MRI compared with CT was similar between groups. However, the early intrahepatic recurrence rate for patients who underwent hepatic resection was significantly higher in ECA-MRI (28.6\%) than in Gd-EOB-MRI (11.6\%) $(p=0.031)$.

Several meta-analyses have described the diagnostic performance of Gd-EOB-MRI for the detection of CRLM $[19,20]$. Chen et al. [19] reported that the pooled weighted sensitivity of Gd-EOB-MRI for detecting liver metastases was 93\% (95\% CI, 90 to 95). In a recent meta-analysis, Gd-EOB-MRI showed an excellent per-lesion sensitivity of $91.2 \%$ (95\% CI, 83.7 to 95.4) for detecting CRLM [20]. Several meta-analyses investigating the diagnostic performance of ECA-MRI were published in 2010 [12,13]. Specifically, Floriani et al. [13] reported that the per-lesion sensitivity of ECA-MRI for detecting
CLRM ranged from $81.9 \%$ to $91.3 \%$, while another metaanalysis demonstrated that the per-lesion mean sensitivity of ECA-MRI was 79.8\% (95\% CI, 62.6 to 90.3) [12]. The results of those studies suggest that Gd-EOB-MRI is better than ECA-MRI for detecting CRLM. However, those meta-analyses were primarily based on retrospective studies, and no direct comparison between Gd-EOB-MRI and ECA-MRI was conducted in any of the original studies included in the metaanalyses. Therefore, an intra-individual study comparing Gd-EOB-MRI and ECA-MRI is necessary to obtain more robust results [19].

To the best of our knowledge, only one intra-individual study prospectively compared Gd-EOB-MRI and ECA-MRI for staging CRLM, and the results of this study confirmed the higher efficacy of Gd-EOB-MRI based on the reduced need for additional preoperative examination and fewer intraoperative plan modifications [9]. Thus, we hypothesized that Gd-EOB-MRI would be superior to ECA-MRI for diagnosing CRLM. Although Gd-EOB-MRI was performed more recently with advanced MR techniques, the diagnostic performance of Gd-EOB-MRI was not significantly different from that of ECA-MRI in our study. There are several possible explanations for these findings. First, with the exception of the hepatobiliary phase, ECA-MRI and Gd-EOB-MRI share most sequences. As MR interpretation in daily practice encompasses all these sequences, the overall diagnostic performance could be similar between the two MRI modalities. Second, the image analysis conducted in the present study was based on a clinical report. According to the previous study [21], initial radiology reports tended to have better diagnostic performance than blinded expert interpretation. The suggested hypothesis was that patient history and clinical data as well as imaging findings might have been considered at the time of the initial reports [21]. Similarly, the clinical information provided at the time of the MR interpretation in our study may show the higher effectiveness of both ECA-MRI and Gd-EOB-MRI. Finally, this study included only liver MRI scans performed before treatment such as chemotherapy. After neoadjuvant chemotherapy, detection and characterization of hepatic lesions become more challenging because of the changes in treated lesions, as well as in response to background parenchymal changes such as steatohepatitis or sinusoidal obstruction syndrome $[22,23]$. Accordingly, further study is needed to compare the diagnostic efficacy of ECA-MRI and Gd-EOB-MRI in the posttreatment setting.

Early recurrence after CRLM resection has been associated with a poor patient prognosis following hepatectomy [24-27]. Therefore, reduction of the early recurrence rate is important to improve patient outcomes and avoid unnecessary hepatic resection. Several predictive factors for early recurrence of CRLM have been identified $[24,25,28]$. According to a recent 
study of 6,025 patients, T3-4 primary tumors, synchronous CRLM, > 3 CRLM, $0 \mathrm{~mm}$ margin liver resection, and associated intraoperative radiofrequency ablation were independent risk factors for early recurrence [24]. Other factors that correlated with higher early recurrence rates include poor differentiation of the primary tumor, bilobar metastases, multiple metastases $(\geq 8)$, microscopic positive surgical margin, and elevated CEA (> $15 \mathrm{ng} / \mathrm{mL}$ ) [25-27]. Early recurrence can be either a regrowth of undetected micrometastasis or true recurrence after complete metastasectomy; therefore, preoperative radiologic evaluation may affect the early recurrence rate. However, the effects of the MRI contrast agent on early recurrence have never been studied. We assumed that the hepatobiliary phase of Gd-EOB-MRI could better detect subclinical metastasis than ECA-MRI, which is supported by our results. The early intrahepatic recurrence rate was lower in the Gd-EOB-MRI group than the ECA-MRI group for patients who underwent hepatic resection, as well as for those considered to have no hepatic metastasis on preoperative MRI, although statistical analysis was unavailable in the latter group. Furthermore, the type of MRI contrast agent was an independent predictor of early intrahepatic recurrence in our study. Gd-EOB-MRI could detect more numbers of tiny metastases that could be missed on ECAMRI, which could lead to a more complete resection of subclinical metastases in the Gd-EOB-MRI group.

In the present study, ECA-MRI and Gd-EOB-MRI showed discordant results in terms of diagnostic performance and early intrahepatic recurrence rate. To accurately evaluate diagnostic performance, an unbiased estimate of the test's accuracy is needed [29]. Specifically, the true disease status of each patient should be determined independently from the imaging test results. However, in clinical practice, patients with a negative test result may not have undergone a gold-standard treatment such as invasive surgery [29]; therefore, the estimated diagnostic performance of tests may be biased [30]. From this perspective, the difference in early recurrence between Gd-EOB-MRI and ECA-MRI may reflect the diagnostic accuracy more realistically, and thus could be more meaningful than a simple comparison of diagnostic performance.

It should be noted that this study has several limitations. First, there was an inevitable selection bias owing to the retrospective cohort design. Second, ECA-MRI and Gd-EOBMRI were performed in different patients at different periods. Unapparent time-dependent biases such as changes in surgical techniques or adjuvant chemotherapeutic regimen may have affected our results. In addition, although most MR protocols were similar between MRI modalities, diffusion-weighted imaging (DWI) was primarily included in the Gd-EOB-MRI group, while only a small portion of ECA-MRI group contained DWI. Nevertheless, the ECA-MRI and Gd-EOB-MRI showed comparable diagnostic performance for evaluating CRLM, which suggests that DWI might not affect the diagnostic performance in our study. Third, the patients that were operated on in the ECA-MRI group had more metastases than those in the Gd-EOB-MRI group. Although the lesion number was not a significant factor on univariate and multivariate analyses, the different lesion number between groups is one of the limitations that must be considered when interpreting our results. Finally, most of the benign lesions were not pathologically confirmed. However, the median follow-up period was long enough to confirm the benignity of the lesions.

In conclusion, Gd-EOB-MRI can potentially decrease the early intrahepatic recurrence rate compared with ECA-MRI after hepatic resection, although the two MRI modalities showed comparable diagnostic performance in colorectal cancer patients. Further prospective studies comparing the survival outcome between Gd-EOB-MRI and ECA-MRI should follow.

\section{Conflicts of Interest}

Conflict of interest relevant to this article was not reported.

\section{References}

1. Manfredi S, Lepage C, Hatem C, Coatmeur O, Faivre J, Bouvier AM. Epidemiology and management of liver metastases from colorectal cancer. Ann Surg. 2006;244:254-9.

2. Norstein J, Silen W. Natural history of liver metastases from colorectal carcinoma. J Gastrointest Surg. 1997;1:398-407.

3. Pawlik TM, Choti MA. Surgical therapy for colorectal metastases to the liver. J Gastrointest Surg. 2007;11:1057-77.

4. Vigano L, Russolillo N, Ferrero A, Langella S, Sperti E, Capus- sotti L. Evolution of long-term outcome of liver resection for colorectal metastases: analysis of actual 5-year survival rates over two decades. Ann Surg Oncol. 2012;19:2035-44.

5. National Comprehensive Cancer Network. Clinical practice guidelines in oncology: colon cancer [Internet]. Fort Washington, PA: National Comprehensive Cancer Network; 2014 [cited 2015 Oct 21]. Available from: http://www.nccn.org/ professionals / physician_gls/pdf/colon.pdf. 
6. National Comprehensive Cancer Network. Clinical practice guidelines in oncology: rectal cancer [Internet]. Fort Washington, PA: National Comprehensive Cancer Network; 2014 [cited 2015 Oct 21]. Available from: http://www.nccn.org/ professionals / physicial_gls/pdf/rectal.pdf.

7. Tirumani SH, Kim KW, Nishino M, Howard SA, Krajewski $\mathrm{KM}$, Jagannathan JP, et al. Update on the role of imaging in management of metastatic colorectal cancer. Radiographics. 2014;34:1908-28.

8. Kulemann V, Schima W, Tamandl D, Kaczirek K, Gruenberger T, Wrba F, et al. Preoperative detection of colorectal liver metastases in fatty liver: MDCT or MRI? Eur J Radiol. 2011;79: e1-6.

9. Zech CJ, Korpraphong P, Huppertz A, Denecke T, Kim MJ, Tanomkiat $\mathrm{W}$, et al. Randomized multicentre trial of gadoxetic acid-enhanced MRI versus conventional MRI or CT in the staging of colorectal cancer liver metastases. Br J Surg. 2014;101:613-21.

10. Han K, Park SH, Kim KW, Kim HJ, Lee SS, Kim JC, et al. Use of liver magnetic resonance imaging after standard staging abdominopelvic computed tomography to evaluate newly diagnosed colorectal cancer patients. Ann Surg. 2015;261: 480-6.

11. Kim HJ, Lee SS, Byun JH, Kim JC, Yu CS, Park SH, et al. Incremental value of liver MR imaging in patients with potentially curable colorectal hepatic metastasis detected at CT: a prospective comparison of diffusion-weighted imaging, gadoxetic acid-enhanced MR imaging, and a combination of both MR techniques. Radiology. 2015;274:712-22.

12. Niekel MC, Bipat S, Stoker J. Diagnostic imaging of colorectal liver metastases with CT, MR imaging, FDG PET, and / or FDG PET/CT: a meta-analysis of prospective studies including patients who have not previously undergone treatment. Radiology. 2010;257:674-84.

13. Floriani I, Torri V, Rulli E, Garavaglia D, Compagnoni A, Salvolini L, et al. Performance of imaging modalities in diagnosis of liver metastases from colorectal cancer: a systematic review and meta-analysis. J Magn Reson Imaging. 2010;31: 19-31.

14. Jhaveri K, Cleary S, Audet P, Balaa F, Bhayana D, Burak K, et al. Consensus statements from a multidisciplinary expert panel on the utilization and application of a liver-specific MRI contrast agent (gadoxetic acid). AJR Am J Roentgenol. 2015; 204:498-509.

15. Merkle EM, Zech CJ, Bartolozzi C, Bashir MR, Ba-Ssalamah A, Huppertz A, et al. Consensus report from the 7th International Forum for Liver Magnetic Resonance Imaging. Eur Radiol. 2016;26:674-82.

16. Kim YK, Kim CS, Han YM, Park G. Detection of small hepatocellular carcinoma: can gadoxetic acid-enhanced magnetic resonance imaging replace combining gadopentetate dimeg- lumine-enhanced and superparamagnetic iron oxide-enhanced magnetic resonance imaging? Invest Radiol. 2010;45:7406.

17. Gray RJ. A class of K-sample tests for comparing the cumulative incidence of a competing risk. Ann Stat. 1988;16:1141-54.

18. Fine JP, Gray RJ. A proportional hazards model for the subdistribution of a competing risk. J Am Stat Assoc. 1999;94: 496-509.

19. Chen L, Zhang J, Zhang L, Bao J, Liu C, Xia Y, et al. Metaanalysis of gadoxetic acid disodium (Gd-EOB-DTPA)-enhanced magnetic resonance imaging for the detection of liver metastases. PLoS One. 2012;7:e48681.

20. Vilgrain V, Esvan M, Ronot M, Caumont-Prim A, Aube C, Chatellier G. A meta-analysis of diffusion-weighted and gadoxetic acid-enhanced MR imaging for the detection of liver metastases. Eur Radiol. 2016;26:4595-615.

21. Mueller GC, Hussain HK, Carlos RC, Nghiem HV, Francis IR. Effectiveness of MR imaging in characterizing small hepatic lesions: routine versus expert interpretation. AJR Am J Roentgenol. 2003;180:673-80.

22. Pilgrim $\mathrm{CH}$, Thomson BN, Banting S, Phillips WA, Michael M. The developing clinical problem of chemotherapy-induced hepatic injury. ANZ J Surg. 2012;82:23-9.

23. Robinson PJ. The effects of cancer chemotherapy on liver imaging. Eur Radiol. 2009;19:1752-62.

24. Vigano L, Capussotti L, Lapointe R, Barroso E, Hubert C, Giuliante F, et al. Early recurrence after liver resection for colorectal metastases: risk factors, prognosis, and treatment. A LiverMetSurvey-based study of 6,025 patients. Ann Surg Oncol. 2014;21:1276-86.

25. Takahashi S, Konishi M, Kinoshita T, Gotohda N, Kato Y, Saito $\mathrm{N}$, et al. Predictors for early recurrence after hepatectomy for initially unresectable colorectal liver metastasis. J Gastrointest Surg. 2013;17:939-48.

26. Malik HZ, Gomez D, Wong V, Al-Mukthar A, Toogood GJ, Lodge JP, et al. Predictors of early disease recurrence following hepatic resection for colorectal cancer metastasis. Eur J Surg Oncol. 2007;33:1003-9.

27. Takahashi S, Konishi M, Nakagohri T, Gotohda N, Saito N, Kinoshita T. Short time to recurrence after hepatic resection correlates with poor prognosis in colorectal hepatic metastasis. Jpn J Clin Oncol. 2006;36:368-75.

28. Tan MC, Butte JM, Gonen M, Kemeny N, Fong Y, Allen PJ, et al. Prognostic significance of early recurrence: a conditional survival analysis in patients with resected colorectal liver metastasis. HPB (Oxford). 2013;15:803-13.

29. Zhou XH. Correcting for verification bias in studies of a diagnostic test's accuracy. Stat Methods Med Res. 1998;7:337-53.

30. Begg CB, Greenes RA. Assessment of diagnostic tests when disease verification is subject to selection bias. Biometrics. 1983;39:207-15. 